\title{
Test cell for electric strength of rubber-epoxy interfaces
}

\author{
Cecilia Forssén, Anna Christerson, Daniel Borg \\ ABB AB Corporate Research \\ Västerås, Sweden \\ cecilia.forssen@se.abb.com
}

\begin{abstract}
Interfaces between solid insulating materials are critical in high voltage apparatus. Here a new test cell for electric strength of rubber-epoxy interfaces was designed and evaluated experimentally. The electrically active part of the interface was about $10 \mathrm{~mm}$ long and the interface endpoints were screened. The rubber in the test cell was compressed and the pressure at the rubber-epoxy interface could be varied and measured. The test cell was designed for AC breakdown testing and in 36 out of 39 tested samples the breakdown occurred at the interface.
\end{abstract}

Keywords-interface; electric strength; breakdown; testing; rubber; epoxy

\section{INTRODUCTION}

Electrically stressed interfaces between solid insulating materials are critical in high voltage apparatus like e.g. cable accessories and connectors. To secure sufficient margins in product designs it is important to study interfacial electric strength and the impact of parameters like contact pressure, surface roughness, lubricants, adhesion between materials, etc.

Measuring electric withstand strength of interfaces between solid insulating materials is not trivial and has been the subject of a CIGRE working group (WG15-10 1996 [1]). This working group among other things recommended that no metal electrodes should be in contact with the interface to prevent undesirable influence from the electrodes on the discharge activity at the interface. In addition the electric field component tangential to the interface should be as uniform as possible and should contribute more to breakdown than the normal field component. Some of the model specimens discussed by the working group are summarized in [2].

There are different types of test cells for electric strength of interfaces presented in the literature. For testing of interfaces with insulating rubber several authors have used spherical or pointed electrodes that were cast into the rubber [1][3][4][5]. These test cells suffer from a common problem with breakdowns occurring in the bulk rubber instead of at the interface. In [6] the spherical electrodes were instead cast into the epoxy which seems to have prevented the problem with breakdowns in the bulk insulation instead of at the interface. However one drawback with the test cell in [6] is the relatively complicated sample manufacturing. Other authors have used plane parallel electrodes and a plastic cylinder with a surrounding rubber ring [7]. With this test cell the interface is in contact with the metallic electrodes and the interface endpoints are highly electrically stressed which is undesirable.

The authors of the current work have previously presented a test cell for testing of interfaces between insulating rubber and silicone gel [8]. That test cell mainly comprised two discshaped electrodes with a rubber cylinder pressed between them. The electrodes and the rubber cylinder were cast in silicone gel thus creating the rubber-gel interface. The test cell worked well: in 14 out of 19 tested samples (AC) the breakdown occurred at the rubber-gel interface. One disadvantage with that test cell was that the mechanical contact pressure at the interface could not be controlled. However mechanical pressure was not of major interest for the rubbersilicone gel interface.

In this work a new test cell for electric strength of rubberepoxy interfaces was designed. The test cell comprised a conical rubber plug that was fitted into a hole in an epoxy disc. The epoxy disc was pressed between two disc-shaped electrodes. The electrically active part of the interface was about $10 \mathrm{~mm}$ long and the interface endpoints were screened. The rubber in the test cell was compressed and the pressure was controlled through a plunger and a spring assembly. The pressure in the rubber-epoxy interface could be measured using a pressure sensor. The test cell was designed for AC breakdown testing.

The major advantage with this new test cell is that the pressure at the interface can be varied and measured. In addition the interface endpoints are electrically screened which prevents discharge initiation from the electrodes and also prevents influence of geometrical defects at the interface endpoints. Moreover the electrodes are not cast in the rubber/epoxy which makes sample manufacturing relatively easy and facilitates for testing of relatively large number of samples. The test cell can be used also for other combinations of solid materials and the length of the interface can be altered. In these cases the detailed electrode shape might need to be adjusted. One drawback with the new test cell is that the field at the interface is non-uniform, although mainly tangential, with a peak value located around the interface midpoint.

The test cell is described in Section II. The sample preparation and breakdown testing are described in Section III. In Section IV the performance of the test cell is briefly evaluated. 


\section{TEST CELL DESIGN}

The test cell was designed for AC breakdown testing of rubber-epoxy interfaces with compressed rubber and with controlled pressure at the interface.

\section{A. Geometry}

The test cell comprised a conical rubber plug that was fitted into a hole in an epoxy disc as shown in Fig. 1. The epoxy disc was pressed between disc shaped electrodes. The pressure at the rubber-epoxy interface was obtained through a plunger that was pressed into the rubber plug. The rubber cylinder had height $20 \mathrm{~mm}$ and small diameter $25 \mathrm{~mm}$. The test cell was kept together by a mechanical support and gel and oil were used to avoid flashover. High voltage was applied to the upper electrodes and ground to the lower electrodes. Fig. 2 shows a schematic axi-symmetric drawing with measures of the inner part of the test cell. The cell was cylinder symmetric around the axis $r=0$ except for the gel container and the mechanical support which were square. The size of the gel container was width $240 \mathrm{~mm}$ and height $125 \mathrm{~mm}$. The radial distance between the epoxy disc and the vertical mechanically supporting rod was $20 \mathrm{~mm}$. The reason for using gel and not oil in the inner part of the cell was to avoid oil penetration into the rubber-epoxy interface. Fig. 3 shows a photo of the mounted test cell without the gel container and the gel. The mechanical support was made of POM (polyoxymetheylene), the gel container was made of transparent polycarbonate, and the gel was a two-component silicone gel.

\section{B. Electrodes}

The inner electrodes extended into the epoxy and were designed to screen the interface endpoints where the interface is in contact with the electrodes. In this way a higher field was obtained at the interface than anywhere else in the rubber thus minimizing the risk of having breakdowns in the bulk rubber instead of at the interface. The protruding parts (marked in blue in Fig. 2) of the inner electrodes were Rogowski-shaped to

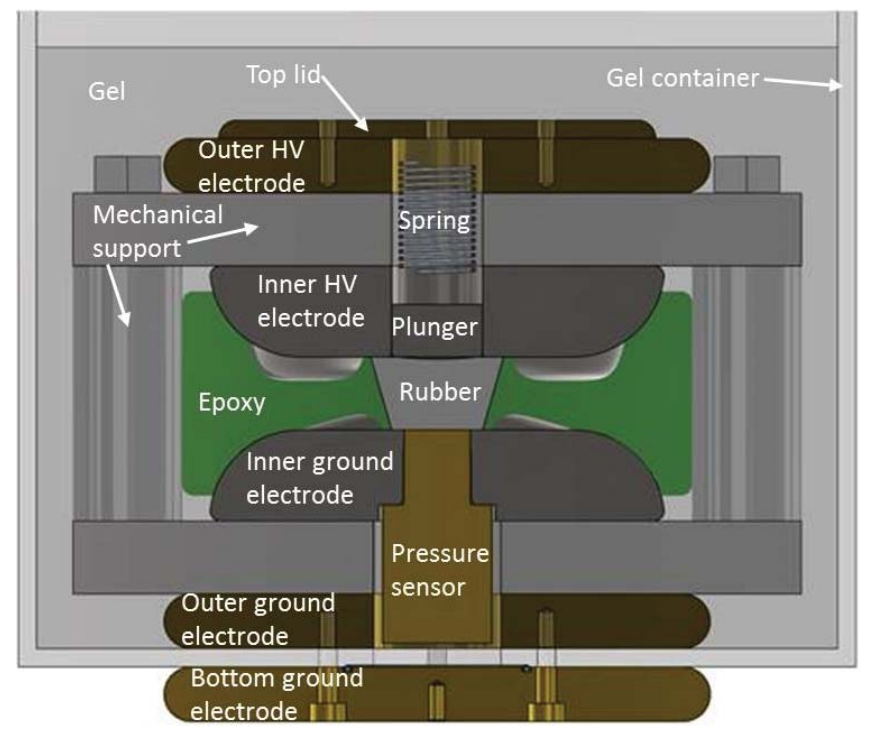

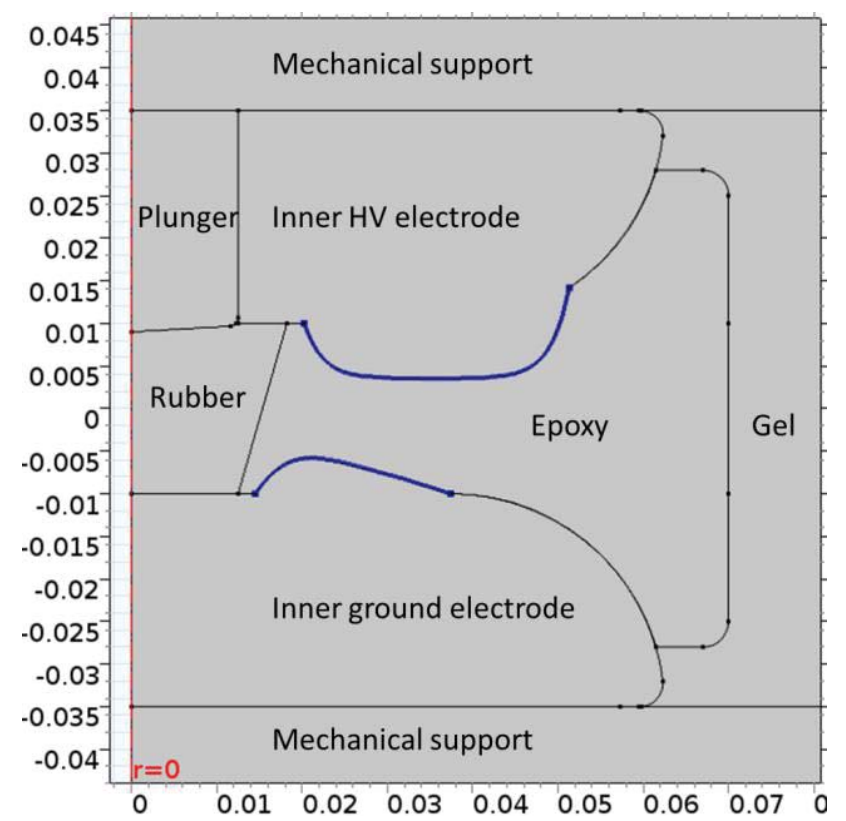

Fig. 2. Schematic drawing of inner part of test cell. Cylindrically symmetric around $r=0$. Measures in meter. Protruding Rogowski-shaped parts of electrodes marked in blue.

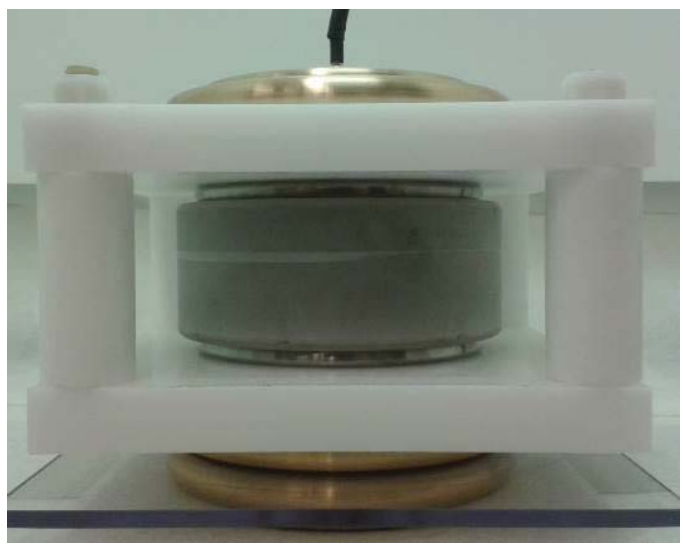

Fig. 3. Photo of mounted test cell without gel container and gel.

avoid local field enhancements at their edges. The protrusions were shifted apart radially to have the same radial distance to the interface and the lower protrusion was rotated clockwise to relax the field in the bulk epoxy. The inner electrodes were made of stainless steel except for the protruding parts which were created through silver painting of the shaped surface of the epoxy disc. A schematic picture of the epoxy disc is shown in Fig. 4. The top inner electrode had a center hole with diameter $25 \mathrm{~mm}$ to allow the spring-loaded plunger to put pressure on the rubber plug. The bottom inner electrode had a center hole for the pressure sensor. The purpose of the outer electrodes was to lower the field in the region where the inner electrode edge is in contact with the mechanical support where there would otherwise be a risk of having breakdown along the gel-POM interface. The outer electrodes were made of brass and had diameter $150 \mathrm{~mm}$, height $15 \mathrm{~mm}$ and corner radius 7.5 $\mathrm{mm}$.

Fig. 1. Schematic picture of test cell without oil volume. 


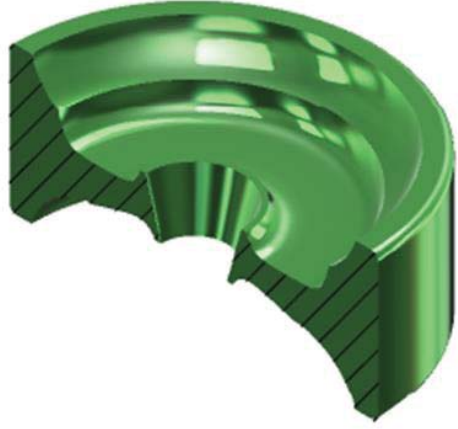

Fig. 4. Schematic picture of epoxy disc.

\section{Pressure}

The pressure at the rubber-epoxy interface was obtained through a stainless steel plunger that was pressed into the rubber plug by use of a spring and a top lid as shown in Fig. 1. The plunger had diameter $25 \mathrm{~mm}$ and a main diameter of 100 $\mathrm{mm}$ to prevent trapping of air between the plunger and the rubber plug. By using different spacers between the spring and the plunger, the interface pressures could be varied. The pressure at the interface is hydrostatic and was measured at the bottom of the rubber plug with a pressure sensor placed inside the lower inner electrode. During breakdown testing the pressure sensor was substituted with a dummy.

\section{Electric field distribution}

The simulated electric field distribution in the inner part of the test cell is shown in Fig. 5. The field is highest in the epoxy in the region with the smallest distance between the protruding parts of the inner electrodes. The Rogowski-shape of the protruding electrode parts prevents local field enhancements at their edges. The field distribution along the rubber-epoxy interface is shown in Fig. 6. The field is mainly tangential. It has a peak value located around the interface midpoint $(z=0)$ and falls off towards the interface endpoints $(\mathrm{z}= \pm 0.01 \mathrm{~m})$ as intended. The tangential field at the interface endpoints is less than $50 \%$ of the peak value which means that the interface endpoints are generally electrically screened. The tangential field is high over a rather long distance along the interface; 5 $\mathrm{mm}$ away from the location of the peak value the field is only reduced with $20 \%$. Based on this it was considered that the electrically active part of the interface was at least $10 \mathrm{~mm}$ long.

\section{EXPERIMENTAL}

\section{A. Mounting of test cell}

All pieces of the test cell were wiped off with ethanol before mounting. The part of the epoxy disc surface that formed the inner electrode surface was painted with silver paint, see Fig. 7. Small pieces of copper foil were placed on the painted surface to obtain good electrical contact with the metal electrodes. Care was taken to avoid air entrapment between the rubber plug and the plunger. Only well-polished electrodes were used. The two components of the silicone gel were first degassed separately for $30 \mathrm{~min}$ at 0.1 bar. After mixing the gel was degassed for another $30 \mathrm{~min}$ at 0.1 bar. The gel was cured in room atmosphere.

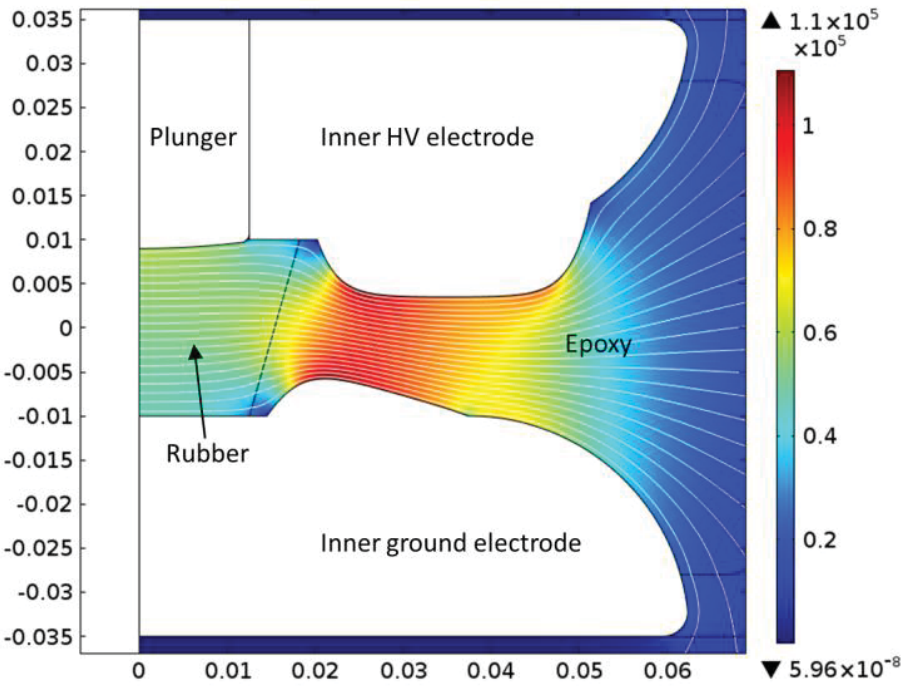

Fig. 5. Simulated electric field distribution (color) and electric equipotential lines. Applied voltage $1 \mathrm{kV}$ peak. Plunger extending $1 \mathrm{~mm}$ into rubber plug.

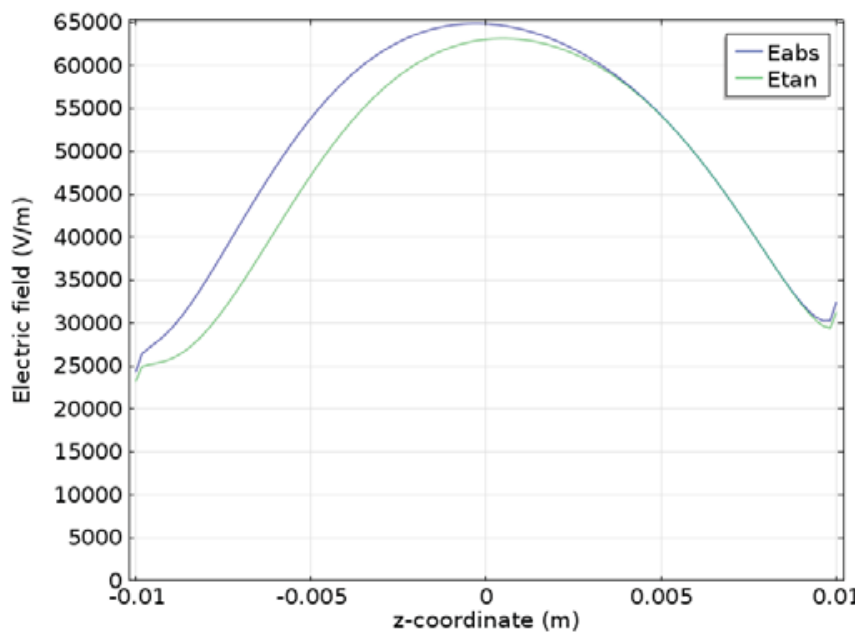

Fig. 6. Simulated electric field in rubber-epoxy interface at applied voltage 1 $\mathrm{kV}$ peak. Absolute field (Eabs) and tangential field (Etan).

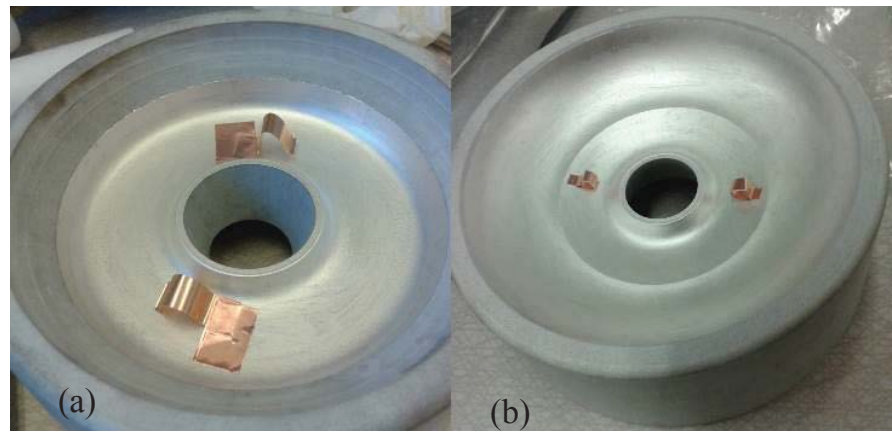

Fig. 7. Surface of epoxy disc with silver paint and copper foil used for contacting (a) high voltage side, (b) ground side.

\section{B. Breakdown testing}

During testing the test cell was partly immersed in transformer oil to prevent flashover. An AC voltage was applied to the test cell and increased stepwise until breakdown occurred. The test 
procedure was set according to Section 9.4 in [9], which is $60 \mathrm{~s}$ step-by-step test.

\section{MEASUREMENT RESULTS}

The test cell was used for AC breakdown measurements on 39 samples. In 36 out of these the breakdown occurred at the rubber-epoxy interface. In two samples the breakdown occurred in the high-field region in the bulk epoxy. In the remaining one sample the breakdown occurred in the bulk rubber and a visible air cavity which probably caused the breakdown was found inside the rubber plug after dismounting. Fig. 8 shows some photos of breakdown tracks after testing.

Breakdown voltage was measured for two different rubbers (A and B) at two different pressures (low and high) and for two different roughness (rough and smooth) of the epoxy surface. The normalized measurement results are presented as a Weibull plot in Fig. 9. A higher electric strength of the interface was obtained for higher pressure and for smoother
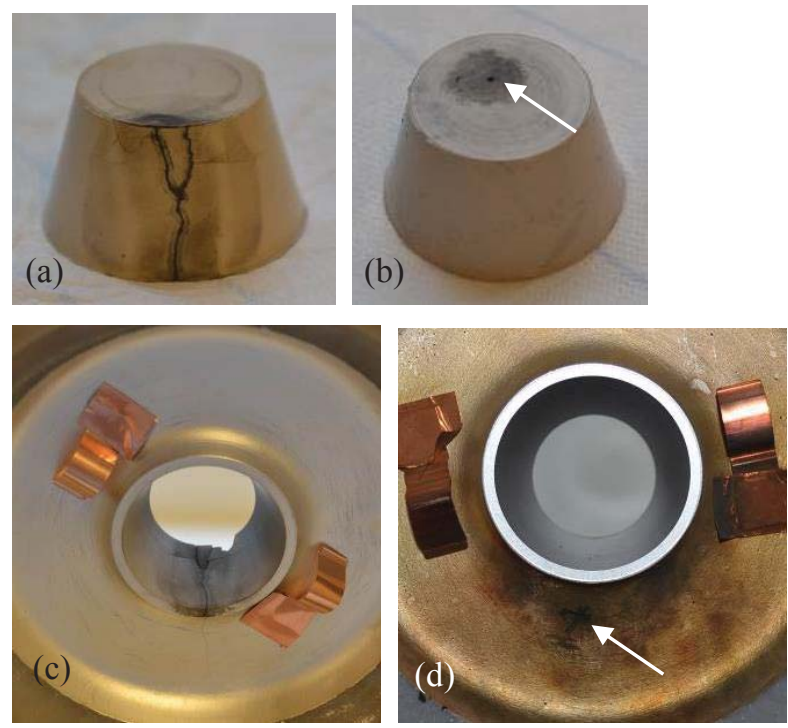

Fig. 8. Photos of breakdown tracks (a) rubber plug after breakdown at interface, (b) breakdown in rubber plug, (c) epoxy disc after breakdown at interface, (d) breakdown in epoxy disc.

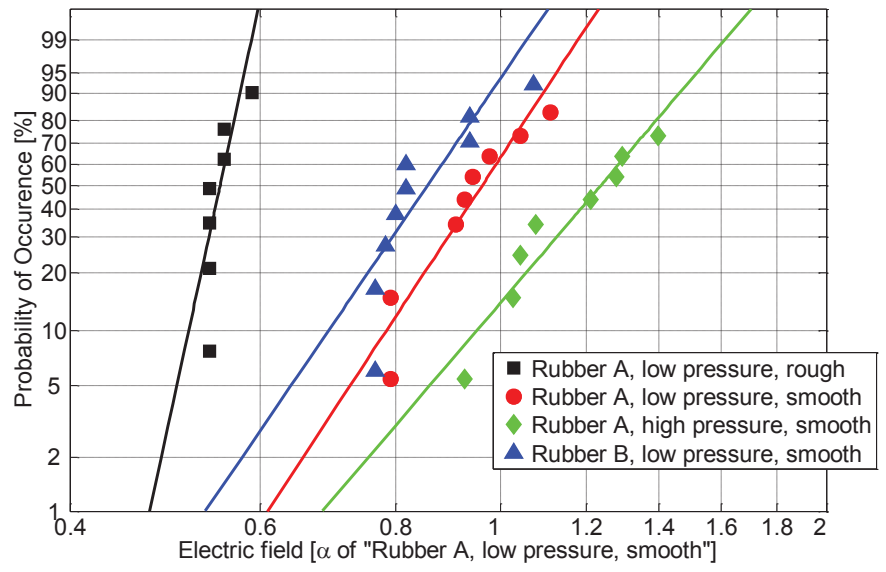

Fig. 9. Weibull plot of breakdown measurement results normalized by Weibull scale parameter $(\alpha)$ of curve "Rubber A, low pressure, smooth". epoxy surface, as expected. The scatter in the data was smaller for the rougher surface than for the smoother surfaces.

\section{CONCLUSION}

A new test cell for electric strength of rubber-epoxy interfaces was designed. The active part of the interface was about 10 $\mathrm{mm}$ long and the interface endpoints were electrically screened. The rubber in the test cell was compressed and the pressure at the rubber-epoxy interface could be varied and measured. The new test cell worked well: in 36 out of 39 tested samples (AC) the breakdown occurred at the rubberepoxy interface. A higher electric strength of the interface was obtained for higher pressure and for smoother epoxy surface, as expected.

The test cell fulfilled the recommendations from the CIRGRE working group WG15-10 [1] except that the electrodes were in contact with the interface. However the interface endpoints were electrically screened to less than $50 \%$ of the tangential peak field which should prevent influence from the electrodes on the discharge activity at the interface.

\section{ACKNOWLEDGMENT}

The authors thank Uno Gäfvert, Jan-Eric Lethagen, and Per Lindholm at ABB Corporate Research, Sweden, Radim Lichy, Fredrik Axelsson and Peter Kjellsson, ABB Kabeldon, Sweden, and Victoria Maurer, ABB Micafil, Switzerland, for valuable support.

\section{REFERENCES}

[1] J. Svahn, L. Hedman and S.M. Gubanski, "Development of Cell for Testing of the Interfacial Electric Strength", CIGRE WG15-10Gubanski-98.

[2] T. Tanaka, M. Nagao, Y. Takahashi, A. Miyazaki, M. Okada, Y. Yamashita, "Dielectric Characteristics of Interfaces in Prefabricated Joints of Extra-high Voltage XLPE Cables", CIGRE Session 2000 Paper 15-206.

[3] Toshihiro Takahashi, Tatsuki Okamoto, Yoshimichi Ohki and Kohei Shibata, "Breakdown Strength at the Interface between Epoxy Resin and Silicone Rubber - A Basic Study for the Development of All Solid Insulation", IEEE Trans. Dielectrics and Electrical Insulation, Vol. 12, No. 4 August 2005, pp. 719-724.

[4] M. Nagao, N Hozumi, Y Muramoto, F.B.C. Pasan, T. Suzuki, T. Tanaka, "Model specimens for testing interfacial electrical insulating properties in EHV extruded cable splices and preliminary results", Proc. Conf. Electrical Insulation and Dielectric Phenomena (CEIDP), 2000, vol.2, pp. 820-3.

[5] J. Höglund and J. Ljungholm, "Investigation of electrical breakdown strength of interfaces", Diploma work, Chalmers University of Technology, Sweden, 2009.

[6] Y.-C. Oh, "Dielectric strength of macro interface between epoxy and rubber according to the interface condition", Transactions of the Korean Institute of Electrical Engineers, C Dec. 2006, vol.55, no.12, pp. 580-5.

[7] D. Kunze, B. Parmigiani, R. Schroth, E. Gockenbach, "Macroscopic internal interfaces in high voltage cable accessories", CIGRE Session 2000 Paper 15-203.

[8] C. Forssén, A. Christerson, D. Borg,"Test cell for interfacial electric strength testing", Proc. IEEE Int. Conf. Solid Dielectrics (ICSD), 2013, pp. 619-622.

[9] IEC 60243-1 Electrical strength of insulating materials - Test methods, Part 1: Tests at power frequency. 